\title{
Double almost statistical convergence of order $\alpha$
}

Ekrem Savaş*

${ }^{*}$ Correspondence:

ekremsavas@yahoo.com

Department of Mathematics, Istanbul Commerce University,

Üsküdar, Istanbul, Turkey

\begin{abstract}
The goal of this paper is to define and study $\lambda$-double almost statistical convergence of order $\alpha$. Further some inclusion relations are examined. We also introduce a new sequence space by combining the double almost statistical convergence and an Orlicz function.
\end{abstract}

MSC: Primary 40B05; secondary 40C05

Keywords: statistical convergence; Orlicz function; double statistical convergence of order $\alpha$; double almost statistical convergence

\section{Introduction}

The notion of statistical convergence was introduced by Fast [1] and Schoenberg [2] independently. Over the years and under different names, statistical convergence was discussed in the theory of Fourier analysis, ergodic theory and number theory. Later on it was further investigated from the sequence space point of view and linked with summability theory by Fridy [3], Connor [4], Šalát [5], Cakalli [6], Miller [7], Maddox [8] and many others. However, Mursaleen [9] defined the concept of $\lambda$-statistical convergence as a new method and found its relation to statistical convergence, $(C, 1)$-summability and strong $(V, \lambda)$-summability. Recently, for $\alpha \in(0,1]$, Çolak and Bektaş [10] have introduced the $\lambda$-statistical convergence of order $\alpha$ and strong $(V, \lambda)$-summability of order $\alpha$ for sequences of complex numbers.

In this paper we define and study $\lambda$-double almost statistical convergence of order $\alpha$. Also, some inclusion relations have been examined.

The notion of statistical convergence depends on the density of subsets of $\mathbf{N}$. A subset $E$ of $\mathbf{N}$ is said to have density $\delta(E)$ if

$$
\delta(E)=\lim _{n \rightarrow \infty} \frac{1}{n} \sum_{k=1}^{n} \chi_{E}(k) \quad \text { exists. }
$$

Note that if $K \subset \mathbf{N}$ is a finite set, then $\delta(K)=0$, and for any set $K \subset \mathbf{N}, \delta\left(K^{C}\right)=1-\delta(K)$.

Definition 1.1 A sequence $x=\left(x_{k}\right)$ is said to be statistically convergent to $\ell$ if for every $\varepsilon>0$,

$$
\delta\left(\left\{k \in \mathbf{N}:\left|x_{k}-\ell\right| \geq \varepsilon\right\}\right)=0 .
$$

We write $s t-\lim x_{k}=L$ in case $x=\left(x_{k}\right)$ is st-statistically convergent to $L$. 
Let $w_{2}$ be the set of all real or complex double sequences. By the convergence of a double sequence we mean the convergence in the Pringsheim sense, that is, the double sequence $x=\left(x_{i j}\right)_{i, j=0}^{\infty}$ has a Pringsheim limit $L$ denoted by $P$-lim $x=L$ provided that, given $\epsilon>0$, there exists $N \in \mathbb{N}$ such that $\left|x_{i j}-L\right|<\epsilon$ whenever $i, j \geq N$. We will describe such an $x$ more briefly as ' $P$-convergent' (see [11]).

We denote by $c_{2}$ the space of $P$-convergent sequences. A double sequence $x=\left(x_{i j}\right)$ is bounded if $\|x\|=\sup _{i, j \geq 0}\left|x_{i j}\right|<\infty$. Let $l_{2}^{\infty}$ and $c_{2}^{\infty}$ be the set of all real or complex bounded double sequences and the set of bounded and convergent double sequences, respectively. Móricz and Rhoades [12] defined the almost convergence of the double sequence as follows: $x=\left(x_{i j}\right)$ is said to be almost convergent to a number $L$ if

$$
P-\lim _{p, q \rightarrow \infty} \sup _{m, n}\left|\frac{1}{(p+1)(q+1)} \sum_{i=m}^{m+p} \sum_{j=n}^{n+q} x_{i j}-L\right|=0,
$$

that is, the average value of $\left(x_{i, j}\right)$ taken over any rectangle

$$
D=\{(i, j): m \leq i \leq m+p, n \leq j \leq n+q\}
$$

tends to $L$ as both $p$ and $q$ tend to $\infty$, and this convergence is uniform in $m$ and $n$. We denote the space of almost convergent double sequences by $\hat{c}_{2}$ as

$$
\hat{c}_{2}=\left\{x=\left(x_{i, j}\right): \lim _{k, l \rightarrow \infty}\left|t_{k l p q}(x)-L\right|=0 \text {, uniformly in } p, q\right\},
$$

where

$$
t_{k l p q}(x)=\frac{1}{(k+1)(l+1)} \sum_{i=p}^{k+p} \sum_{j=q}^{l+q} x_{i, j}
$$

The notion of almost convergence for single sequences was introduced by Lorentz [13] and some others.

A double sequence $x$ is called strongly double almost convergent to a number $L$ if

$$
P-\lim _{k, l \rightarrow \infty} \frac{1}{(k+1)(l+1)} \sum_{i=p}^{k+p} \sum_{j=q}^{l+q}\left|x_{i, j}-L\right|=0 \quad \text { uniformly in } p, q \text {. }
$$

By $\left[\hat{c}_{2}\right]$ we denote the space of strongly almost convergent double sequences. It is easy to see that the inclusions $c_{2}^{\infty} \subset\left[\hat{c}_{2}\right] \subset \hat{c}_{2} \subset l_{2}^{\infty}$ strictly hold.

The notion of strong almost convergence for single sequences has been introduced by Maddox [8].

A linear functional $L$ on $l_{2}^{\infty}$ is said to be a Banach limit if it has the following properties:

(1) $L(x) \geq 0$ if $x \geq 0$ (i.e., $x_{i, j} \geq 0$ for all $i, j$ ),

(2) $L(e)=1$, where $e=\left(e_{i, j}\right)$ with $e_{i, j}=1$ for all $i, j$ and

(3) $L(x)=L\left(S_{10} x\right)=L\left(S_{01} x\right)=L\left(S_{11} x\right)$, where the shift operators $S_{10} x, S_{01} x, S_{11} x$ are defined by $S_{10} x=\left(x_{i+1, j}\right), S_{01} x=\left(x_{i, j+1}\right), S_{11} x=\left(x_{i+1, j+1}\right)$.

Let $B_{2}$ be the set of all Banach limits on $l_{2}^{\infty}$. A double sequence $x=\left(x_{i, j}\right)$ is said to be almost convergent to a number $L$ if $L(x)=L$ for all $L \in B_{2}$ (see [14]). 
The idea of statistical convergence was extended to double sequences by Mursaleen and Edely [15]. More recent developments on double sequences can be found in [16-18], where some more references can be found. For the single sequences, statistical convergence of order $\alpha$ and strong $p$-Cesàro summability of order $\alpha$ was introduced by Çolak [19]. Quite recently, in [10], Çolak and Bektaş generalized this notion by using de la Valée-Poussin mean.

Let $K \subseteq \mathbb{N} \times \mathbb{N}$ be a two-dimensional set of positive integers and let $K_{m, n}$ be the numbers of $(i, j)$ in $K$ such that $i \leq n$ and $j \leq m$.

Then the lower asymptotic density of $K$ is defined as

$$
P-\liminf _{m, n} \frac{K_{m, n}}{m n}=\delta_{2}(K) .
$$

In the case when the sequence $\left(\frac{K_{m, n}}{m n}\right)_{m, n=1,1}^{\infty, \infty}$ has a limit, we say that $K$ has a natural density and is defined as

$$
P-\lim _{m, n} \frac{K_{m, n}}{m n}=\delta_{2}(K) .
$$

For example, let $K=\left\{\left(i^{2}, j^{2}\right): i, j \in \mathbb{N}\right\}$, where $\mathbb{N}$ is the set of natural numbers. Then

$$
\delta_{2}(K)=P-\lim _{m, n} \frac{K_{m, n}}{m n} \leq P-\lim _{m, n} \frac{\sqrt{m} \sqrt{n}}{m n}=0
$$

(i.e., the set $K$ has double natural density zero).

Mursaleen and Edely [15] presented the notion statistical convergence for a double sequence $x=\left(x_{i j}\right)$ as follows: A real double sequence $x=\left(x_{i j}\right)$ is said to be statistically convergent to $L$ provided that for each $\epsilon>0$,

$$
P-\lim _{m, n} \frac{1}{m n} \mid\left\{(i, j): i \leq m \text { and } j \leq n,\left|x_{i j}-L\right| \geq \epsilon\right\} \mid=0 .
$$

We now give the following definition.

The double statistical convergence of order $\alpha$ is defined as follows. Let $0<\alpha \leq 1$ be given. The sequence $\left(x_{i j}\right)$ is said to be statistically convergent of order $\alpha$ if there is a real number $L$ such that

$$
P-\lim _{m n \rightarrow \infty} \frac{1}{(m n)^{\alpha}} \mid\left\{i \leq m \text { and } j \leq n:\left|x_{i j}-L\right| \geq \epsilon\right\} \mid=0
$$

for every $\epsilon>0$, in which case we say that $x$ is double statistically convergent of order $\alpha$ to $L$. In this case, we write $S_{2}^{\alpha}-\lim x_{i j}=L$. The set of all double statistically convergent sequences of order $\alpha$ will be denoted by $S_{2}^{\alpha}$. If we take $\alpha=1$ in this definition, we can have previous definition.

Let $\lambda=\left(\lambda_{n}\right)$ be a non-decreasing sequence of positive numbers tending to $\infty$ such that

$$
\lambda_{n+1} \leq \lambda_{n}+1, \quad \lambda_{1}=1 .
$$

The generalized de la Valèe-Poussin mean is defined by

$$
t_{n}(x)=\frac{1}{\lambda_{n}} \sum_{k \in I_{n}} x_{k}
$$


where $I_{n}=\left[n-\lambda_{n}+1, n\right]$. A sequence $x=\left(x_{n}\right)$ is said to be $(V, \lambda)$-summable to a number $L$ if $t_{n}(x) \rightarrow L$ as $n \rightarrow \infty$.

In [9] Mursaleen introduced the idea of $\lambda$-statistical convergence for a single sequence as follows:

The number sequence $x=\left(x_{i}\right)$ is said to be $\lambda$-statistically convergent to the number $\ell$ if for each $\epsilon>0$,

$$
\lim _{n} \frac{1}{\lambda_{n}}\left|\left\{n-\lambda_{n}+1 \leq i \leq n:\left|x_{i}-L\right| \geq \epsilon\right\}\right|=0 .
$$

In this case, we write $S_{\lambda}-\lim _{i} x_{i}=\ell$ and we denote the set of all $\lambda$-statistically convergent sequences by $S_{\lambda}$.

Definition 1.2 Let $\lambda=\left(\lambda_{m}\right)$ and $\mu=\left(\mu_{n}\right)$ be two non-decreasing sequences of positive real numbers both of which tend to $\infty$ as $m$ and $n$ approach $\infty$, respectively. Also, let $\lambda_{m+1} \leq \lambda_{m}+1, \lambda_{1}=1$ and $\mu_{n+1} \leq \mu_{n}+1, \mu_{1}=1$. We write the generalized double de la Valèe-Poussin mean by

$$
t_{m n}(x)=\frac{1}{\lambda_{m} \mu_{n}} \sum_{i \in I_{m}, j \in I_{n}} x_{i j}
$$

A sequence $x=\left(x_{i j}\right)$ is said to be $\left(V^{2}, \lambda, \mu\right)$-summable to a number $L$, if $t_{m n}(x) \rightarrow L$ as $m, n \rightarrow \infty$ in the Pringsheim sense. Throughout this paper, we denote $\bar{\lambda}_{m n}$ by $\lambda_{m} \mu_{n}$ and $\left(i \in I_{m}, j \in I_{n}\right)$ by $(i, j) \in I_{m n}$.

\section{Main results}

In this section, we define $\lambda$-double almost statistically convergent sequences of order $\alpha$. Also, we prove some inclusion theorems.

We now have the following.

Definition 2.1 Let $0<\alpha \leq 1$ be given. The sequence $x=\left(x_{i j}\right) \in w_{2}$ is said to be $\hat{S}_{\bar{\lambda}}^{\alpha}$ statistically convergent of order $\alpha$ if there is a real number $L$ such that

$$
P-\lim _{m n \rightarrow \infty} \frac{1}{\bar{\lambda}_{m n}^{\alpha}}\left|\left\{(k, l) \in I_{m n}:\left|t_{k l p q}(x)-L\right| \geq \epsilon\right\}\right|=0 \quad \text { uniformly in } p, q,
$$

where $\bar{\lambda}_{m n}^{\alpha}$ denotes the $\alpha$ th power $\left(\bar{\lambda}_{m n}\right)^{\alpha}$ of $\bar{\lambda}_{m n}$. In case $x=\left(x_{i j}\right)$ is $\hat{S}_{\bar{\lambda}}^{\alpha}$-statistically convergent of order $\alpha$ to $L$, we write $\hat{S}_{\bar{\lambda}}^{\alpha}-\lim x_{i j}=L$. We denote the set of all $\hat{S}_{\bar{\lambda}}^{\alpha}$-statistically convergent sequences of order $\alpha$ by $\hat{S}_{\bar{\lambda}}^{\alpha}$. We write $\hat{S}_{2}$ if $\bar{\lambda}_{m n}=m n$ and $\alpha=1$ for $\hat{S}_{\bar{\lambda}}^{\alpha}$.

We know that the $\hat{S}_{\bar{\lambda}}^{\alpha}$-statistical convergence of order $\alpha$ is well defined for $0<\alpha \leq 1$, but it is not well defined for $\alpha>1$ in general. For this let $x=\left(x_{i j}\right)$ be fixed. Then, for an arbitrary number $L$ and $\epsilon>0$, we write

$$
\begin{gathered}
P-\lim _{m n \rightarrow \infty} \frac{1}{\overline{\bar{\lambda}}_{m n}^{\alpha}}\left|\left\{(k, l) \in I_{m n}:\left|t_{k l p q}(x)-L\right| \geq \epsilon\right\}\right| \\
\quad \leq \lim _{n \rightarrow \infty} \frac{\left[\bar{\lambda}_{m n}\right]+1}{\bar{\lambda}_{m n}^{\alpha}}=0 \quad \text { uniformly in } p, q .
\end{gathered}
$$

Therefore $\hat{S}_{\bar{\lambda}}^{\alpha}-\lim x_{i j}$ is not uniquely determined for $\alpha>1$. 
Definition 2.2 Let $0<\alpha \leq 1$ be any real number and let $r$ be a positive real number. A sequence $x$ is said to be strongly $\hat{w}_{r}^{\alpha}(\bar{\lambda})$-summable of order $\alpha$, if there is a real number $L$ such that

$$
P-\lim _{m n \rightarrow \infty} \frac{1}{\bar{\lambda}_{m n}^{\alpha}} \sum_{(k, l) \in I_{m n}}\left|t_{k l p q}(x)-L\right|^{r}=0 \quad \text { uniformly in } p, q .
$$

If we take $\alpha=1$, the strong $\hat{w}_{r}^{\alpha}(\bar{\lambda})$-summability of order $\alpha$ reduces to the strong $\hat{w}_{r}(\bar{\lambda})$ summability.

We denote the set of all strongly $\hat{w}_{r}^{\alpha}(\bar{\lambda})$-summable sequences of order $\alpha$ by $\hat{w}_{p}^{\alpha}(\bar{\lambda})$.

We now are ready to state the following theorem.

Theorem 2.1 If $0<\alpha \leq \beta \leq 1$, then $\hat{S}_{\bar{\lambda}}^{\alpha} \subset \hat{S}_{\bar{\lambda}}^{\beta}$.

Proof Let $0<\alpha \leq \beta \leq 1$. Then

$$
\frac{1}{\bar{\lambda}_{m n}^{\beta}}\left|\left\{(k, l) \in I_{m n}:\left|t_{k l p q}(x)-L\right| \geq \epsilon\right\}\right| \leq \frac{1}{\bar{\lambda}_{m n}^{\alpha}}\left|\left\{(k, l) \in I_{m n}:\left|t_{k l p q}(x)-L\right| \geq \epsilon\right\}\right|
$$

for every $\epsilon>0$, and finally we have that $\hat{S}_{\bar{\lambda}}^{\alpha} \subset \hat{S}_{\bar{\lambda}}^{\beta}$. This proves the result.

We have the following from the previous theorem.

\section{Corollary 2.1}

(i) If a sequence is $\hat{S}_{\bar{\lambda}}^{\alpha}$-statistically convergent of order $\alpha$ to L, then it is $\hat{S}_{\bar{\lambda}}$-statistically convergent to $L$, that is, $\hat{S}_{\bar{\lambda}}^{\alpha} \subset \hat{S}_{\bar{\lambda}}$ for each $\alpha \in(0,1]$,

(ii) $\alpha=\beta \Longrightarrow \hat{S}_{\bar{\lambda}}^{\alpha}=\hat{S}_{\bar{\lambda}}^{\beta}$,

(iii) $\alpha=1 \Longrightarrow \hat{S}_{\bar{\lambda}}^{\alpha}=\hat{S}_{\bar{\lambda}}$.

Theorem 2.2 $\hat{S}_{2}^{\alpha} \subseteq \hat{S}_{\bar{\lambda}}^{\alpha}$ if

$$
\lim _{m n \rightarrow \infty} \inf \frac{\bar{\lambda}_{m n}^{\alpha}}{(m n)^{\alpha}}>0
$$

Proof For given $\epsilon>0$, we write

$$
\left\{k \leq m \text { and } l \leq m:\left|t_{k l p q}(x)-L\right| \geq \epsilon\right\} \supset\left\{(k, l) \in I_{m n}:\left|t_{k l p q}(x)-L\right| \geq \epsilon\right\}
$$

and so

$$
\begin{aligned}
& \frac{1}{(m n)^{\alpha}} \mid\left\{k \leq m \text { and } l \leq m:\left|t_{k l p q}(x)-L\right| \geq \epsilon\right\} \mid \\
& \quad \geq \frac{\bar{\lambda}_{m n}^{\alpha}}{(m n)^{\alpha}} \frac{1}{\bar{\lambda}_{m n}^{\alpha}}\left|\left\{(k, l) \in I_{m n}:\left|t_{k l p q}(x)-L\right| \geq \epsilon\right\}\right| .
\end{aligned}
$$

Using (2.1) and taking the limit as $m n \rightarrow \infty$, we have $\hat{S}_{2}^{\alpha}-\lim x_{i j}=L \Longrightarrow \hat{S}_{\bar{\lambda}}^{\alpha}-\lim x_{k l}=L$.

Theorem 2.3 Let $0<\alpha \leq \beta \leq 1$ and $r$ be a positive real number, then $\hat{w}_{r}^{\alpha}(\bar{\lambda}) \subseteq \hat{w}_{r}^{\beta}(\bar{\lambda})$. 
Proof Let $x=\left(x_{i j}\right) \in \hat{w}_{r}^{\alpha}(\bar{\lambda})$. Then given $\alpha$ and $\beta$ such that $0<\alpha \leq \beta \leq 1$ and a positive real number $r$, we write

$$
\frac{1}{\bar{\lambda}_{m n}^{\beta}} \sum_{(k, l) \in I_{m n}}\left|t_{k l p q}(x)-L\right|^{r}
$$

and we get that $\hat{w}_{r}^{\alpha}(\bar{\lambda}) \subseteq \hat{w}_{r}^{\beta}(\bar{\lambda})$.

We have the following corollary which is a consequence of Theorem 2.3.

Corollary 2.2 Let $0<\alpha \leq \beta \leq 1$ and $r$ be a positive real number. Then

(i) If $\alpha=\beta$, then $\hat{w}_{r}^{\alpha}(\bar{\lambda})=\hat{w}_{r}^{\beta}(\lambda)$.

(ii) $\hat{w}_{r}^{\alpha}(\bar{\lambda}) \subseteq \hat{w}_{r}(\bar{\lambda})$ for each $\alpha \in(0,1]$ and $0<r<\infty$.

Theorem 2.4 Let $\alpha$ and $\beta$ be fixed real numbers such that $0<\alpha \leq \beta \leq 1$ and $0<r<\infty$. If a sequence is a strongly $\hat{w}_{r}^{\alpha}(\bar{\lambda})$-summable sequence of order $\alpha$ to L, then it is $\hat{S}_{\bar{\lambda}}^{\beta}$-statistically convergent of order $\beta$ to L, i.e., $\hat{w}_{r}^{\alpha}(\bar{\lambda}) \subset \hat{S}_{\bar{\lambda}}^{\beta}$.

Proof For any sequence $x=\left(x_{i j}\right)$ and $\epsilon>0$, we write

$$
\begin{aligned}
\sum_{(k, l) \in I_{m n}}\left|t_{k l p q}(x)-L\right|^{r} & =\sum_{\substack{(k, l) \in I_{m n} \\
\left|t_{k l p q}(x)-L\right| \geq \epsilon}}\left|t_{k l p q}(x)-L\right|^{r}+\sum_{\substack{(k, l) \in I_{m n} \\
\left|t_{k l p q}(x)-L\right|<\epsilon}}\left|t_{k l p q}(x)-L\right|^{r} \\
& \geq \sum_{\substack{(k, l) \in I_{m n} \\
\left|t_{k l p q}(x)-L\right| \geq \epsilon}}\left|t_{k l p q}(x)-L\right|^{p} \geq\left|\left\{(k, l) \in I_{m n}:\left|t_{k l p q}(x)-L\right| \geq \epsilon\right\}\right| \cdot \epsilon^{r}
\end{aligned}
$$

and so that

$$
\begin{aligned}
\frac{1}{\overline{\bar{\lambda}}_{m n}^{\alpha}} \sum_{(k, l) \in I_{m n}}\left|t_{k l p q}(x)-L\right|^{r} & \geq \frac{1}{\bar{\lambda}_{m n}^{\alpha}}\left|\left\{(k, l) \in I_{m n}:\left|t_{k l p q}(x)-L\right| \geq \epsilon\right\}\right| \cdot \epsilon^{r} \\
& \geq \frac{1}{\bar{\lambda}_{m n}^{\beta}}\left|\left\{(k, l) \in I_{m n}:\left|t_{k l p q}(x)-L\right| \geq \epsilon\right\}\right| \cdot \epsilon^{r} .
\end{aligned}
$$

This shows that if $x=\left(x_{i j}\right)$ is a strongly $\hat{w}_{r}^{\alpha}(\bar{\lambda})$-summable sequence of order $\alpha$ to $L$, then it is $\hat{S}_{\bar{\lambda}}^{\beta}$-statistically convergent of order $\beta$ to $L$. This completes the proof.

We have the following corollary.

Corollary 2.3 Let $\alpha$ be fixed real numbers such that $0<\alpha \leq 1$ and $0<r<\infty$.

(i) If a sequence is a strongly $\hat{w}_{r}^{\alpha}(\bar{\lambda})$-summable sequence of order $\alpha$ to L, then it is $\hat{S}_{\bar{\lambda}}^{\alpha}$-statistically convergent of order $\alpha$ to L, i.e., $\hat{w}_{r}^{\alpha}(\bar{\lambda}) \subset \hat{S}_{\bar{\lambda}}^{\alpha}$.

(ii) $\hat{w}_{r}^{\alpha}(\bar{\lambda}) \subset \hat{S}_{\bar{\lambda}}$ for $0<\alpha \leq 1$.

\section{Some sequence spaces}

In present section, we study the inclusion relations between the set of $\hat{S}_{\bar{\lambda}}^{\alpha}$-statistically convergent sequences of order $\alpha$ and strongly $\hat{w}_{r}^{\alpha}[\bar{\lambda}, M]$-summable sequences of order $\alpha$ with respect to an Orlicz function $M$. 
Recall in [20] that an Orlicz function $M:[0, \infty) \rightarrow[0, \infty)$ is continuous, convex, nondecreasing function such that $M(0)=0$ and $M(x)>0$ for $x>0$, and $M(x) \rightarrow \infty$ as $x \rightarrow \infty$.

An Orlicz function $M$ is said to satisfy $\Delta_{2}$-condition for all values of $u$ if there exists $K>0$ such that $M(2 u) \leq K M(u), u \geq 0$.

Lindenstrauss and Tzafriri [21] used the idea of an Orlicz function to construct the sequence space

$$
\ell_{M}=\left\{x \in w: \sum_{k=1}^{\infty} M\left(\frac{\left|x_{k}\right|}{\rho}\right)<\infty \text { for some } \rho>0\right\} .
$$

The space $\ell_{M}$ with the norm

$$
\|x\|=\inf \left\{\rho>0: \sum_{k=1}^{\infty} M\left(\frac{\left|x_{k}\right|}{\rho}\right) \leq 1\right\}
$$

becomes a Banach space called an Orlicz sequence space. The space $\ell_{M}$ is closely related to the space $\ell_{p}$ which is an Orlicz sequence space with $M(x)=|x|^{r}$ for $1 \leq r<\infty$.

In the later stage, different classes of Orlicz sequence spaces were introduced and studied by Parashar and Choudhary [22], Savaş [23-28] and many others.

Definition 3.1 Let $M$ be an Orlicz function, $r=\left(r_{k l}\right)$ be a sequence of strictly positive real numbers and let $\alpha \in(0,1]$ be any real number. Now we write

$$
\begin{aligned}
\hat{w}_{r}^{\alpha}[\bar{\lambda}, M]= & \left\{x=\left(x_{k l}\right): P-\lim _{m n \rightarrow \infty} \frac{1}{\overline{\bar{\lambda}}_{m n}^{\alpha}} \sum_{(k, l) \in I_{m n}}\left[\frac{M\left(\left|t_{k l p q}(x)-L\right|\right)}{\rho}\right]^{r_{k l}}=0\right. \\
& \text { uniformly in } p, q \text { for some } L \text { and } \rho>0\} .
\end{aligned}
$$

If $x \in \hat{w}_{r}^{\alpha}[\bar{\lambda}, M]$, then we say that $x$ is almost strongly double $\lambda$-summable of order $\alpha$ with respect to the Orlicz function $M$.

If we consider various assignments of $M, \bar{\lambda}$ and $r$ in the above sequence spaces, we are granted the following:

(1) If $M(x)=x, \bar{\lambda}_{m n}=m n$ and $r_{k, l}=1$ for all $(k, l)$, then $\hat{w}_{r}^{\alpha}[\bar{\lambda}, M]=\left[\hat{w}^{\alpha}\right]$.

(2) If $r_{k, l}=1$ for all $(k, l)$, then $\hat{w}_{r}^{\alpha}[\bar{\lambda}, M]=\hat{w}^{\alpha}[\bar{\lambda}, M]$.

(3) If $r_{k, l}=1$ for all $(k, l)$ and $\bar{\lambda}_{m n}=m n$, then $\hat{w}_{r}^{\alpha}[\bar{\lambda}, M]=\hat{w}^{\alpha}[M]$.

(4) If $\bar{\lambda}_{m n}=m n$, then $\hat{w}_{r}^{\alpha}[\bar{\lambda}, M]=\hat{w}_{r}^{\alpha}[M]$.

We now have the following theorem.

Theorem 3.1 If $r_{k, l}>0$ and $x$ is almost strongly $\lambda$-double convergent to $L_{1}$ with respect to the Orlicz function $M$, that is, $x_{k l} \rightarrow L_{1}\left(\hat{w}_{r}^{\alpha}[\bar{\lambda}, M]\right)$, then $L_{1}$ is unique.

The proof of Theorem 3.1 is straightforward. So, we omit it.

In the following theorems, we assume that $r=\left(r_{k l}\right)$ is bounded and $0<h=\inf _{k l} r_{k l} \leq$ $r_{k l} \leq \sup _{k l} r_{k l}=H<\infty$.

Theorem 3.2 Let $\alpha, \beta \in(0,1]$ be real numbers such that $\alpha \leq \beta$ and $M$ be an Orlicz function, then $\hat{w}_{r}^{\alpha}[\bar{\lambda}, M] \subset \hat{S}_{\bar{\lambda}}^{\beta}$. 
Proof Let $x \in \hat{w}_{r}^{\alpha}[\bar{\lambda}, M], \epsilon>0$ be given and $\sum_{1}$ and $\sum_{2}$ denote the sums over $(k, l) \in I_{m n}$, $\left|t_{k l p q}(x)-L\right| \geq \epsilon$ and $(k, l) \in I_{m n},\left|t_{k l p q}(x)-L\right|<\epsilon$, respectively. Since $\lambda_{m n}^{\alpha} \leq \bar{\lambda}_{m n}^{\beta}$, for each $m, n$ we write

$$
\begin{aligned}
& \frac{1}{\overline{\bar{\lambda}}_{m n}^{\alpha}} \sum_{(k, l) \in I_{m n}}\left[\frac{M\left(\left|t_{k l p q}(x)-L\right|\right)}{\rho}\right]^{r_{k l}} \\
& \quad=\frac{1}{\bar{\lambda}_{m n}^{\alpha}}\left[\sum_{1}\left[\frac{M\left(\left|t_{k l p q}(x)-L\right|\right)}{\rho}\right]^{r_{k l}}+\sum_{2}\left[\frac{M\left(\left|t_{k l p q}(x)-L\right|\right)}{\rho}\right]^{r_{k l}}\right] \\
& \geq \frac{1}{\bar{\lambda}_{m n}^{\beta}}\left[\sum_{1}\left[\frac{M\left(\left|t_{k l p q}(x)-L\right|\right)}{\rho}\right]^{r_{k l}}+\sum_{2}\left[\frac{M\left(\left|t_{k l p q}(x)-L\right|\right)}{\rho}\right]^{r_{k l}}\right] \\
& \geq \frac{1}{\bar{\lambda}_{m n}^{\beta}} \sum_{1}[f(\epsilon)]^{r_{k l}} \\
& \geq \frac{1}{\bar{\lambda}_{m n}^{\beta}} \sum_{1} \min \left(\left[M\left(\epsilon_{1}\right)\right]^{h},\left[M\left(\epsilon_{1}\right)\right]^{H}\right), \quad \epsilon_{1}=\frac{\epsilon}{\rho} \\
& \geq \frac{1}{\bar{\lambda}_{m n}^{\beta}}\left|\left\{(k, l) \in I_{m n}:\left|t_{k l p q}(x)-L\right| \geq \epsilon\right\}\right| \min \left(\left[M\left(\epsilon_{1}\right)\right]^{h},\left[M\left(\epsilon_{1}\right)\right]^{H}\right) .
\end{aligned}
$$

Since $x \in \hat{w}_{r}^{\alpha}[\bar{\lambda}, M]$, the left-hand side of the above inequality tends to zero as $m n \rightarrow \infty$ uniformly in $p, q$. Hence the right-hand side tends to zero as $m n \rightarrow \infty$ uniformly in $p, q$ and therefore $x \in \hat{S}_{\bar{\lambda}}^{\beta}$. This proves the result.

Corollary 3.1 Let $\alpha \in(0,1]$ and $M$ be an Orlicz function, then $\hat{w}_{r}^{\alpha}[\bar{\lambda}, M] \subset \hat{S}_{\bar{\lambda}}^{\alpha}$.

We conclude this paper with the following theorem.

Theorem 3.3 Let $M$ be an Orlicz function and $x=\left(x_{i j}\right)$ be a bounded sequence, then $\hat{S}_{\bar{\lambda}}^{\alpha} \subset$ $\hat{w}_{r}^{\alpha}[\bar{\lambda}, M]$.

Proof Suppose that $x \in \ell_{2}^{\infty}$ and $\hat{S}_{\bar{\lambda}}^{\alpha}-\lim x_{i j}=L$. Since $x \in \ell_{2}^{\infty}$, then there is a constant $T>0$ such that $\left|t_{k l p q}(x)\right| \leq T$. Given $\epsilon>0$, we write for all $p, q$

$$
\begin{aligned}
& \frac{1}{\overline{\bar{\lambda}}_{m n}^{\alpha}} \sum_{(k, l) \in I_{m n}}\left[M\left(\frac{\left|t_{k l p q}(x)-L\right|}{\rho}\right)\right]^{r_{k l}} \\
& =\frac{1}{\bar{\lambda}_{m n}^{\alpha}} \sum_{1}\left[M\left(\frac{\left|t_{k l p q}(x)-L\right|}{\rho}\right)\right]^{r_{k l}}+\frac{1}{\bar{\lambda}_{m n}^{\alpha}} \sum_{2}\left[M\left(\frac{\left|t_{k l p q}(x)-L\right|}{\rho}\right)\right]^{r_{k l}} \\
& \leq \frac{1}{\bar{\lambda}_{m n}^{\alpha}} \sum_{1} \max \left\{\left[M\left(\frac{T}{\rho}\right)\right]^{h},\left[M\left(\frac{T}{\rho}\right)\right]^{H}\right\}+\frac{1}{\bar{\lambda}_{m n}^{\alpha}} \sum_{2}\left[M\left(\frac{\epsilon}{\rho}\right)\right]^{r_{k l}} \\
& \leq \max \left\{[M(K)]^{h},[M(K)]^{H}\right\} \frac{1}{\bar{\lambda}_{m n}^{\alpha}}\left|\left\{(k, l) \in I_{m n}:\left|t_{k l p q}(x)-L\right| \geq \epsilon\right\}\right| \\
& \quad+\max \left\{\left[M\left(\epsilon_{1}\right)\right]^{h},\left[M\left(\epsilon_{1}\right)\right]^{H}\right\}, \quad \frac{T}{\rho}=K, \frac{\epsilon}{\rho}=\epsilon_{1} .
\end{aligned}
$$

Therefore $\hat{w}_{p}^{\alpha}[\bar{\lambda}, M]$. This proves the result. 


\section{Competing interests}

The author declares that they have no competing interests.

Received: 16 November 2012 Accepted: 15 February 2013 Published: 20 March 2013

\section{References}

1. Fast, H: Sur la convergence statistique. Colloq. Math. 2, 241-244 (1951)

2. Schoenberg, IJ: The integrability of certain functions and related summability methods. Am. Math. Mon. 66, 361-375 (1959)

3. Fridy, JA: On statistical convergence. Analysis 5, 301-313 (1985)

4. Connor, J: The statistical and strong p-Cesàro convergence of sequences. Analysis 8, 47-63 (1988)

5. Šalát, T: On statistical convergence of real numbers. Math. Slovaca 30, 139-150 (1980)

6. Cakalli, H: A study on statistical convergence. Funct. Anal. Approx. Comput. 1(2), 19-24 (2009)

7. Miller, HI: A measure theoretical subsequence characterization of statistical convergence. Trans. Am. Math. Soc. 347(5), 1811-1819 (1995)

8. Maddox, IJ: On strong almost convergence. Math. Proc. Camb. Philos. Soc. 85(2), 345-350 (1979)

9. Mursaleen, M: $\lambda$-statistical convergence. Math. Slovaca 50(1), 111-115 (2000)

10. Çolak, R, Bektaş, CA: $\lambda$-statistical convergence of order $\alpha$. Acta Math. Sci., Ser. B 31(3), 953-959 (2011)

11. Pringsheim, A: Zur theorie der zweifach unendlichen Zahlenfolgen. Math. Ann. 53, $289-321$ (1900)

12. Móricz, F, Rhoades, BE: Almost convergence of double sequences and strong regularity of summability matrices. Math. Proc. Camb. Philos. Soc. 104, 283-294 (1988)

13. Lorentz, GG: A contribution to the theory of divergent sequences. Acta Math. 80, 167-190 (1948)

14. Basarir, M, Konca, S: On some lacunary almost convergent double sequence spaces and Banach limits. Abstr. Appl. Anal. 2012, Article ID 426357 (2012)

15. Mursaleen, M, Edely, OH: Statistical convergence of double sequences. J. Math. Anal. Appl. 288(1), 223-231 (2003)

16. Bhunia, S, Das, P, Pal, S: Restricting statistical convergence. Acta Math. Hung. 134(1-2), 153-161 (2012)

17. Cakalli, H, Savaş, E: Statistical convergence of double sequences in topological groups. J. Comput. Anal. Appl. 12(2), 421-426 (2010)

18. Mursaleen, M, Çakan, C, Mohiuddine, SA, Savaş, E: Generalized statistical convergence and statistical core of double sequences. Acta Math. Sin. Engl. Ser. 26(11), 2131-2144 (2010)

19. Çolak, R: Statistical convergence of order $\alpha$. In: Modern Methods in Analysis and Its Applications, pp. 121-129. Anamaya Pub., New Delhi (2010)

20. Krasnosel'skii, MA, Rutisky, YB: Convex Functions and Orlicz Spaces. Noordhoff, Groningen (1961)

21. Lindenstrauss, J, Tzafriri, L: On Orlicz sequence spaces. Isr. J. Math. 101, 379-390 (1971)

22. Parashar, SD, Choudhary, B: Sequence space defined by Orlicz function. Indian J. Pure Appl. Math. 25(14), 419-428 (1994)

23. Savaș, E: $(A, \lambda)$-double sequence spaces defined by Orlicz function and double statistical convergence. Comput. Math. Appl. 55(6), 1293-1301 (2008)

24. Savaş, E, Savaş, R: Some $\lambda$-sequence spaces defined by Orlicz functions. Indian J. Pure Appl. Math. 34(12), 1673-1680 (2003)

25. Savaş, E: On some new double lacunary sequences spaces via Orlicz function. J. Comput. Anal. Appl. 11(3), 423-430 (2009)

26. Savaş, E, Savaş, R: Some sequence spaces defined by Orlicz functions. Arch. Math. 40(1), 33-40 (2004)

27. Savaş, E, Patterson, RF: $(\bar{\lambda}, \sigma)$-double sequence spaces via Orlicz function. J. Comput. Anal. Appl. 10(1), 101-111 (2008)

28. Savaş, E, Patterson, RF: Double sequence spaces defined by Orlicz functions. Iran. J. Sci. Technol., Trans. A, Sci. 31(2), 183-188 (2007)

doi:10.1186/1687-1847-2013-62

Cite this article as: Savaş: Double almost statistical convergence of order $\alpha$. Advances in Difference Equations 2013 2013:62.

\section{Submit your manuscript to a SpringerOpen ${ }^{\ominus}$ journal and benefit from:}

- Convenient online submission

Rigorous peer review

- Immediate publication on acceptance

- Open access: articles freely available online

- High visibility within the field

- Retaining the copyright to your article 